\title{
KERNEL GROWTH PHYSIOLOGY OF WHEAT UNDER LATE PLANTING HEAT STRESS
}

\author{
M. A. HASAN ${ }^{1 *}$ and J. U. AHMED ${ }^{2}$ \\ 1 Department of Crop Botany, Hajee Mohammad Danesh Science and Technology University, Dinajpur, Bangladesh. \\ 2 Department of Crop Botany, Bangabandhu Sheikh Mujibur Rahman Agricultural University, Gazipur, \\ Bangladesh.
}

(Accepted: 26 January 2005)

\begin{abstract}
Three heat tolerant (Aghrani, Kanchan and CB30) and one heat sensitive (Sonora) wheat genotypes were tested under normal and post anthesis heat stress condition by seeding them on November 30 and December 30,2001 at the research farm of Bangabandhu Sheikh Mujibur Rahman Agricultural University (BSMRAU), Gazipur, Bangladesh to study their kernel growth physiology in relation to heat stress. Irrespective of growing conditions, both dry matter and starch accumulation in kernel showed a typical sigmoidal pattern in all genotypes. Due to post anthesis heat stress, the quantity of dry matter accumulation at peak and the duration required to attain peak were higher in heat tolerant ( $\mathrm{H}_{\mathrm{T}}$ ) genotypes than the heat sensitive (HS) one. Sonora, a HS genotype, responded to post anthesis heat stress by accumulating larger quantity of soluble sugar during active kernel development period in contrast to $\mathrm{HT}$ genotypes indicating poor capacity of kernel of Sonora to utilize sugar for starch synthesis. The heat tolerant genotypes exhibited distinctly higher relative individual kernel dry weight under post anthesis heat stress condition when compared to heat sensitive genotype.
\end{abstract}

Key words: heat stress, kernel growth, sugar-starch conversion, wheat.

\section{INTRODUCTION}

In Bangladesh, late planted wheat (Triticum aestivum L.) is exposed to high temperature at the reproductive stage resulting in yield reduction. Both reduction in kernel number per spike $\mathrm{e}^{2,5,11,13}$ and kernel size $\mathrm{e}^{1,3}$ account for the reduction in grain yield. In spite of low yield of wheat due to post anthesis heat stress, cultivation of wheat cannot be avoided totally. Because the irrigation dependent Boro rice cultivation may need to be replaced in future by partially irrigated or non irrigated wheat cultivation to overcome the arsenic problem. Therefore, an effort ought to be made to minimize the late sown yield reduction by screening or developing high temperature tolerant wheat genotypes/ varieties. Membrane thermostability (MT) test is a widely used and acceptable method to evaluate heat tolerance. The wheat genotypes having the ability to withstand high temperature stress are known as heat tolerant $(\mathrm{HT})$ genotypes and they show greater heat killing time in MT test. On the other hand, the genotypes which do not have the ability to withstand high temperature stress are known as heat sensitive genotypes and they show lesser heat killing time in MT test. ${ }^{27}$

Post anthesis heat stress in wheat induces several physiological effects which eventually result in smaller kernel size due to reduced grain filling period ${ }^{12,22}$ and reduced grain filling rate ${ }^{21}$, ${ }^{24}$ or the combined effect of both. ${ }^{23}$ However, the physiological and biochemical mechanisms involved in temperature dependent grain growth are yet to be studied. The present investigation was carried out to study the kernel growth physiology in relation to heat stress.

\section{METHODS AND MATERIALS}

Three heat tolerant (Aghrani, Kanchan and CB30) and one heat sensitive (Sonora) wheat genotypes were tested under normal and post anthesis heat stress condition by seeding them on November 30 and December 30 respectively, at the research farm of Bangabandhu Sheikh Mujibur Rahman Agricultural University (BSMRAU), Gazipur, Bangladesh during November, 2001 to May, 2002 on an upland soil. The experiment was conducted in a split plot design with four replications. The two growing conditions- normal growing condition (sowing on November 30 ) and post anthesis heat stress condition (sowing on December 30 ) were the main plot treatments whereas the four wheat genotypes were placed randomly as sub-plot treatments. Seeds of four wheat genotypes were sown in rows

\footnotetext{
" Corresponding author
} 
$20 \mathrm{~cm}$ apart, at the rate of $120 \mathrm{~kg} / \mathrm{ha}$ in a unit plot size of $3 \mathrm{~m} \times 2 \mathrm{~m}$. A fertilizer dose of 100-38-25-28 $\mathrm{kg} / \mathrm{ha} \mathrm{N}, \mathrm{P}, \mathrm{K}$ and $\mathrm{S}$ was applied in the form of Urea, Triple Super Phosphate (TSP), Muriate of Potash (MP) and Gypsum, respectively. Irrigations were given to avoid the drought stress when necessary. Other intercultural operations were done uniformly.

Kernel growth rate and dry matter accumulation: At anthesis, 70 main shoot spikes were tagged from each plot. Five tagged spikes were harvested to quantify kernel growth at every $4^{\text {th }}$ day beginning from anthesis. The harvesting of spikes in all genotypes was continued up to $40 \mathrm{~d}$ after anthesis (DAA) for normal growing condition (November 30 sowing) and 36 DAA for post anthesis heat stress conditions (December 30 sowing). The harvested spikes were kept in an oven at $70^{\circ} \mathrm{C}$ for $72 \mathrm{~h} .20$ kernels of each genotype were separated from the middle two spikelets of each of the five spikes. During separation, only first and second kernel of a spikelet were collected. The weight of 20 kernels of each genotype was determined with an analytical balance (AND Electronic Balance Model ER 180A A \& D Company Limited, Tokyo, Japan). The samples were stored in desiccators to avoid moisture absorption for sugar and starch analysis.

The absolute kernel growth rate (AGR) was calculated using the following formula:

$A G R=\frac{W_{2}-W_{1}}{T_{2}-T_{1}}$

Where,

$$
\begin{aligned}
& \mathrm{W}_{1}=\text { Kernel dry weight at initial time } \\
& \mathrm{W}_{2}=\text { Kernel dry weight at final time } \\
& \mathrm{T}_{1}=\text { Initial time } \\
& \mathrm{T}_{2}=\text { Final time. }
\end{aligned}
$$

Estimation of soluble sugar and starch: The soluble sugar and starch content of kernel at $4 \mathrm{~d}$ interval from anthesis in case of all wheat genotypes in both growing conditions were determined. ${ }^{26}$

Soluble sugar estimation: The dried composite kernel samples from four replicates were ground finely by mortar and pestle. $50 \mathrm{mg}$ of finely ground sample was placed in a $15 \mathrm{ml}$ centrifuge tube and $10 \mathrm{ml}$ of $80 \%$ ethanol was added. The tubes were covered with aluminium foil and kept in a water bath at $80-85^{\circ} \mathrm{C}$ for $30 \mathrm{~min}$. Then the samples were centrifuged and decanted into a $50-\mathrm{ml}$ beaker. This extraction was repeated three more times. The alcohol extract was evaporated on a water bath at $80-85^{\circ} \mathrm{C}$ until most of the alcohol was removed (the volume was reduced to about $3 \mathrm{ml}$ ). The volume was made up to $25 \mathrm{ml}$ with distilled water.

$5 \mathrm{ml}$ of this sugar extract was transferred to a $100 \mathrm{ml}$ volumetric flask and made up to volume with distilled water. $5 \mathrm{ml}$ of this diluted sugar extract was taken into a pyrex test tube and then these tubes and the tubes containing the glucose standards $(0,1,2,3,4$, and $5 \mathrm{mg} / 5 \mathrm{ml})$ were placed in an ice bath. To each tube $10 \mathrm{ml}$ of anthrone reagent $(2 \mathrm{~g}$ of anthrone dissolved in one liter of concentrated sulfuric acid and stored in a refrigerator; A fresh solution was prepared every 2 d.) was added slowly, allowing the reagent to run down the side of the test tube. The solution was stirred slowly with a glass rod. The tubes were placed in a boiling water bath for exactly $7.5 \mathrm{~min}$ and cooled immediately in ice. The absorbance was measured at $630 \mathrm{~nm}$ and sugar content as $\mathrm{mg} /$ kernel was estimated using a standard curve.

Starch estimation: The residue left in the centrifuge tube after collecting sugar extract was dried in an oven at $80^{\circ} \mathrm{C}$ and $2 \mathrm{ml}$ of distilled water was added to each centrifuge tube. The tubes were placed in a boiling water bath for $15 \mathrm{~min}$ and stirred occasionally. The tubes were allowed to cool and $2 \mathrm{ml}$ of $9.2 \mathrm{~N} \mathrm{HCIO}_{4}$ was added to each tube while stirring constantly. Then the solution was stirred occasionally for $15 \mathrm{~min}$. The suspension was then made up to about $10 \mathrm{ml}$ and centrifuged. The supernatant was collected and 2 $\mathrm{ml}$ of $4.6 \mathrm{~N} \mathrm{HCIO}_{4}$ was added to the residue. This suspension was stirred for $15 \mathrm{~min}$ and was made up to $10 \mathrm{ml}$ with distilled water. Then the suspension was centrifuged and the supernatants were combined. The combined supernatants were made up to $50 \mathrm{ml}$ with distilled water. $5 \mathrm{ml}$ of starch extract was transferred to a $50 \mathrm{ml}$ volumetric flask and made up to volume with distilled water. $5 \mathrm{ml}$ of this diluted starch extract 
was taken into a pyrex test tube. Then these tubes and the tubes containing glucose standards $(0,1$, $2,3,4$, and $5 \mathrm{mg} / 5 \mathrm{ml}$ including $0.6 \mathrm{ml} 0.46 \mathrm{~N}$ $\mathrm{HCIO}_{4}$ solution for each tube) were placed in an ice bath. To each tube $10 \mathrm{ml}$ of the anthrone reagent was added slowly, allowing the reagent to run down the side of the test tube. The solution was stirred slowly with a glass rod. Then tubes were put in a boiling water bath for exactly 7.5 min and were cooled immediately in ice. Then the absorbance was measured at $630 \mathrm{~nm}$ and starch content was estimated as $\mathrm{mg} /$ kernel using standard curve.

No. of kernels/spike: Five spikes from the main shoot were collected randomly per plot and number of kernels per spike was counted manually.

No. of spikes and grain yield $/ \mathrm{m}^{2}$ : The samples were collected from an area of $1 \mathrm{~m} \mathrm{X} 1 \mathrm{~m}$ from the center of each plot by cutting the plant at ground level. Then spikes were counted and collected in a cloth bag ( $\left.2^{\prime} \times 1.5^{\prime}\right)$. The samples were sun dried, threshed and cleaned manually and fresh weight of grain determined. Grain yield were expressed in $\mathrm{t} / \mathrm{ha}$, adjusted to $12 \%$ moisture.

Individual kernel size: From each plot, thousand kernels were taken randomly from the dried sample and weighed. From this weight, average individual kernel size was calculated.

Relative performance: The relative performance was calculated by the following formula ${ }^{4}$ :

$\begin{aligned} & \text { Variable measured under } \\ & \text { Relative } \\ & \text { performance condition }\end{aligned}$
$(\%) \quad \frac{\begin{array}{c}\text { Variable measured under } \\ \text { normal condition }\end{array}}{\text { normal }}$ 100

Temperature: Air temperature was recorded at 2 $h$ interval throughout the experimental period and daily mean temperature was calculated manually.

Statistical analysis: The data were analyzed using MSTAT programme.

\section{RESULTS AND DISCUSSION}

All the wheat genotypes sown in the field on November 30 were exposed to $<26^{\circ} \mathrm{C}$ during the reproductive growth phase which was very close to normal temperature of $22-26^{\circ} \mathrm{C}$ required for reproductive growth ${ }^{2,7}$ and considered as normal growing condition. On the other hand, when sown on December 30, they experienced high temperature $\left(>26^{\circ} \mathrm{C}\right)$ during the reproductive phase and was regarded as post anthesis heat stress (Figure 1).

Kernel dry matter accumulation: Kernel dry matter accumulation followed a more or less similar pattern in all four genotypes both under normal and post anthesis heat stress conditions (Figure 2). A typical sigmoidal pattern of dry matter accumulation was discernible in all genotypes. Under normal growing condition, the dry weight in Aghrani was observed to be increased up to $53.69 \mathrm{mg} / \mathrm{kernel} 36 \mathrm{~d}$ after anthesis (DAA) and declined thereafter slowly. In other two HT genotypes i.e. in Kanchan and CB30 , the dry weight was found to be increased up to $51.35 \& 49.62 \mathrm{mg} /$ kernel, respectively $32 \mathrm{~d}$ after anthesis and declined thereafter. The HS genotype Sonora showed similar pattern and attained the highest dry weight $(53.04 \mathrm{mg} / \mathrm{seed})$ $32 \mathrm{~d}$ after anthesis.

Under post anthesis heat stress condition, maximum dry matter accumulation per kernel and duration to attain that kernel dry matter were reduced in all wheat genotypes. The extent of reduction in dry matter accumulation in heat tolerant Aghrani (9.0\%), Kanchan (8.5\%) and CB$30(6.59 \%)$ was closely followed by slightly higher reduction rate in the HS Sonora (10.25\%). Again, the reduction in duration required to attain the maximum kernel was 4 days in the $\mathrm{HT}$ genotypes whereas it was reduced by 8 days in the Sonora. The declining tendency in dry matter after attaining the highest level could be due to respiratory loss of kernel. Thus it indicates the importance of harvesting the crop and quickly drying the wheat grains when they attain physiological maturity. Sigmoidal pattern of seed dry matter accumulation was also found in wheat. ${ }^{8}$ 


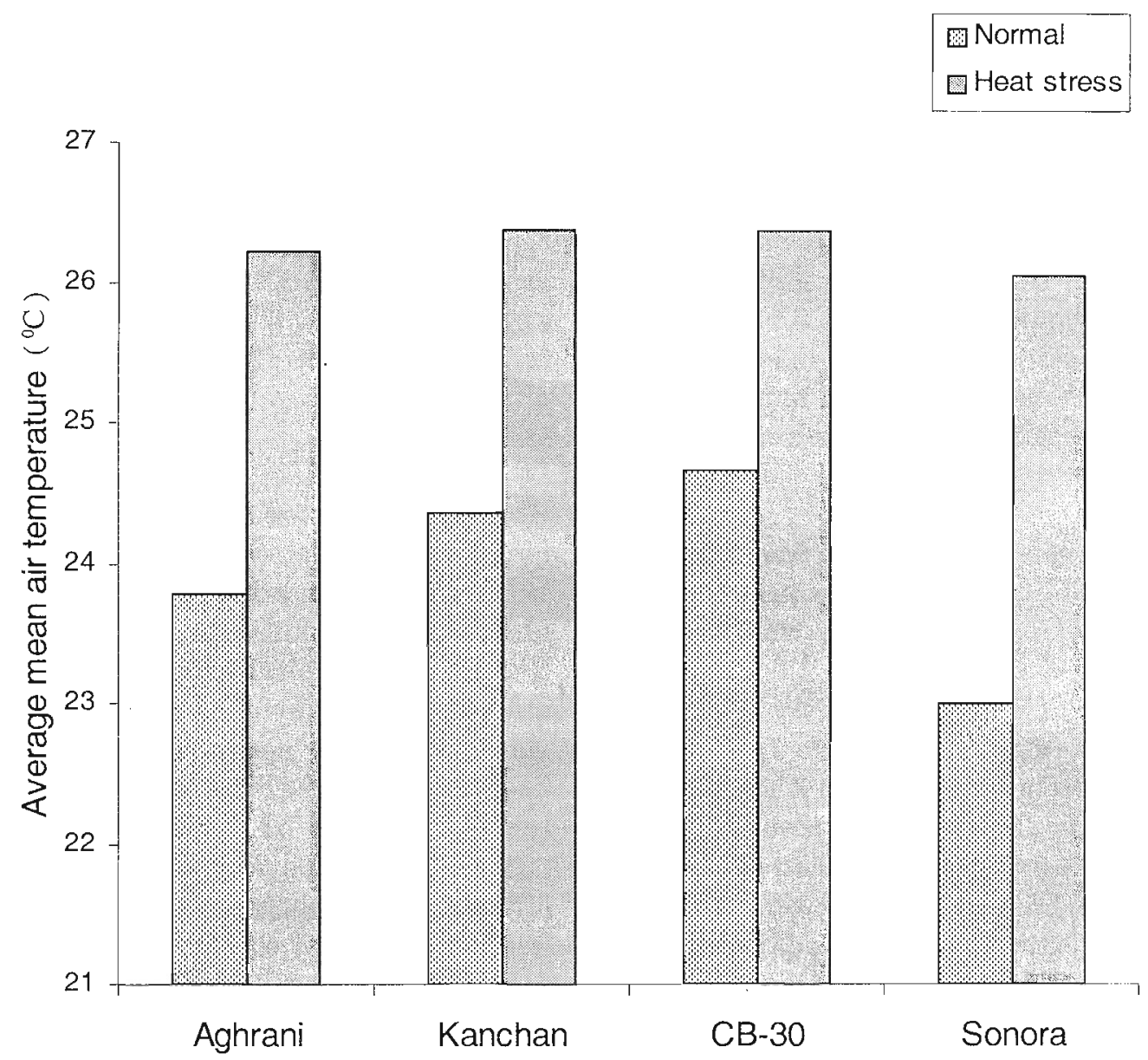

Figure 1: Average mean air temperature $\left({ }^{\circ} \mathrm{C}\right)$ received by different wheat genotypes during anthesis to maturity under normal and post anthesis heat stress conditions

Kernel Growth rate: The absolute growth rate and duration of rapid growth rate (more than 1 $\mathrm{mg} /$ day/kernel) revealed significant differences among the genotypes and between the growing conditions (Figure 3). Under normal growing condition, all the genotypes maintained a longer duration of rapid growth rate, apparently 22-30 days. But under post anthesis heat stress condition, this duration was reduced. The HT genotypes possessed this duration of rapid growth rate of about 16-28 days whereas the HS genotype, Sonora, maintained that growth rate only for 10 days. These results agreed with previous studies. ${ }^{5}$ They found that delayed sowing caused high temperature stress conditons at grain filling, resulting in reduced kernel filling period and rate but the reductions were lower in tolerant varieties than in sensitive varieties. Similar effects have been observed in previous studies. ${ }^{6,15,19,20,25}$
The kernel growth rate data indicate that soon after attaining the highest rate, the plant entered a stage of internal starvation, which caused initiation of declining growth rate. Then grain growth depends largely on the stored food materials mobilized from other plant parts, thus enhancing the whole plant senescence. Under normal condition, all genotypes delayed to initiate the phase of internal starvation and, as a result, had longer durations of rapid growth than those under heat stress. At post anthesis heat stress they initiated the starvation phase earlier and reduced the rapid growth duration. But the HS Sonora because of its earliest cessation of rapid growth rate had minimum kernel growth duration. 

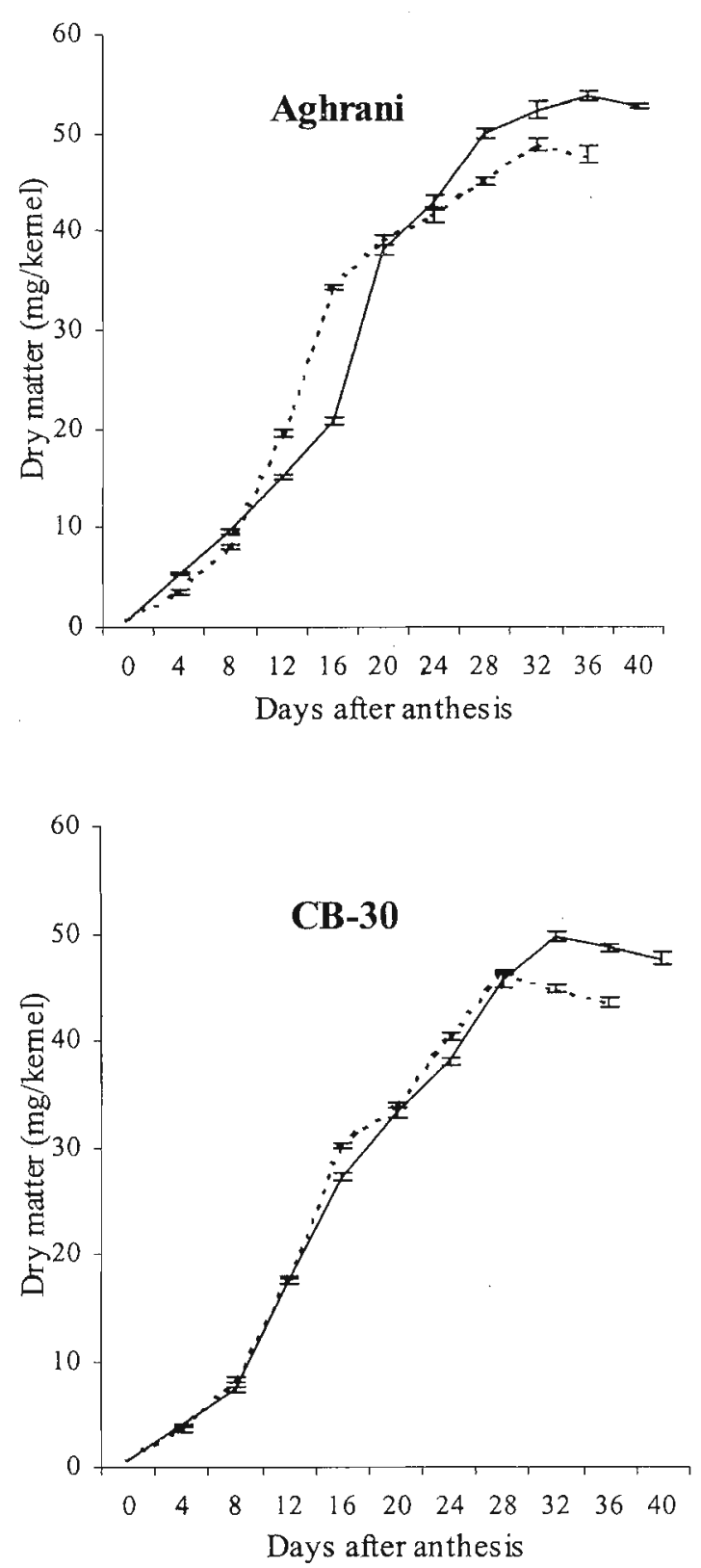
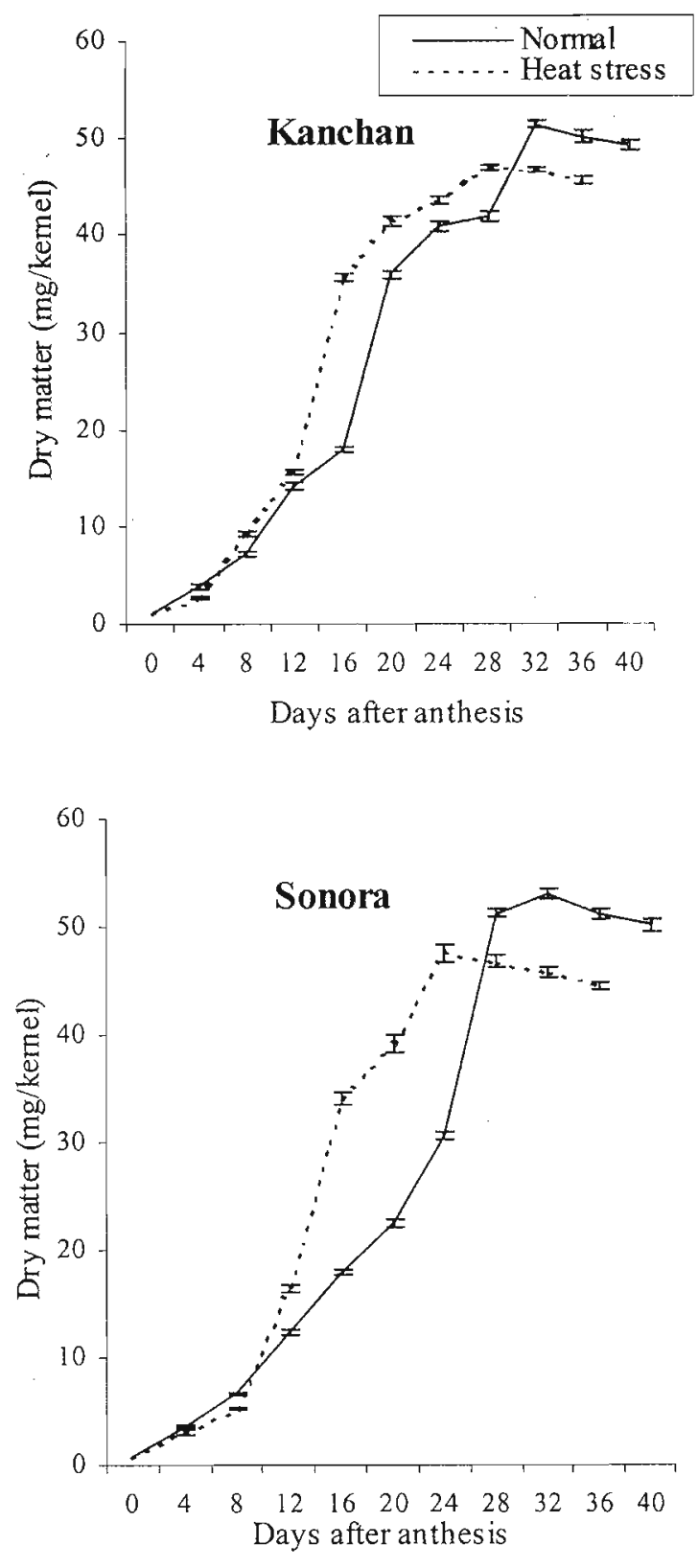

Figure 2: Kernel dry matter accumulation in different wheat genotypes at different days after anthesis under normal and post anthesis heat stress conditions.

Starch accumulation pattern in kernel: Starch contributes up to $74 \%$ of the kernel dry matter and is thus considered as the major contributor to kernel dry matter accumulation during development. In the present experiment, starch accumulation followed a sigmoidal pattern in tested wheat genotypes and in both growing conditions (Figure 4). Under normal growing condition the starch accumulation pattern reached the highest level at 32 days after anthesis (DAA) in HT genotypes, Kanchan and CB-30 and in the HS genotype Sonora. Another HT genotype Aghrani reached the maximum starch accumulation stage at $36 \mathrm{DAA}$. This result revealed that no clear difference between $\mathrm{HT}$ and HS genotypes in respect to duration of maximum starch level under normal condition. But under heat stress condition the data showed not only clear reduction in days to reach the peak level of starch accumulation but also indicated variations 
between HT and HS genotypes. Days to reach the peak starch accumulation were reduced by 4 days in HT genotypes (e.g., Aghrani, Kanchan, CB-30) whereas such reduction was extended to 8 days in HS genotypes. It is clear here that times to reach the maximum dry matter and times to reach the highest starch in kernel coincided and hence the importance of starch in kernel dry matter accumulation became evident. Chanda et al. $(1999)^{8}$ also found a close correspondence between these two parameters.

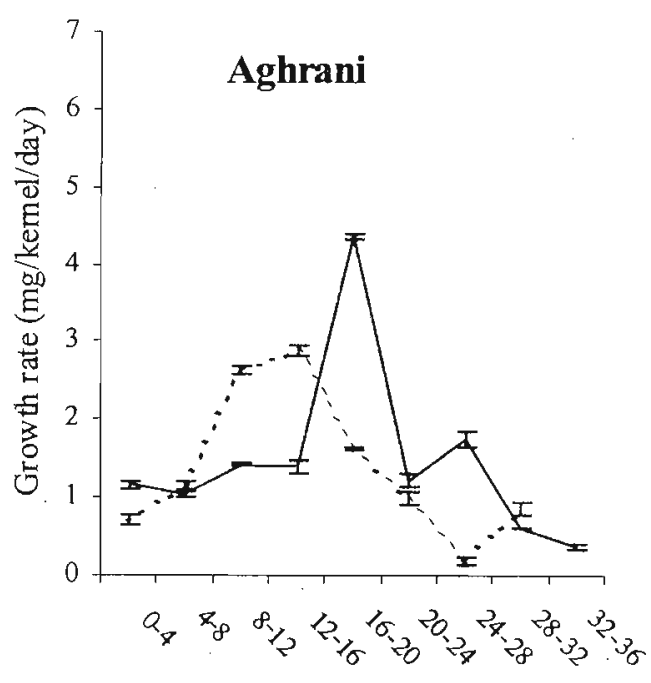

Days after anthesis

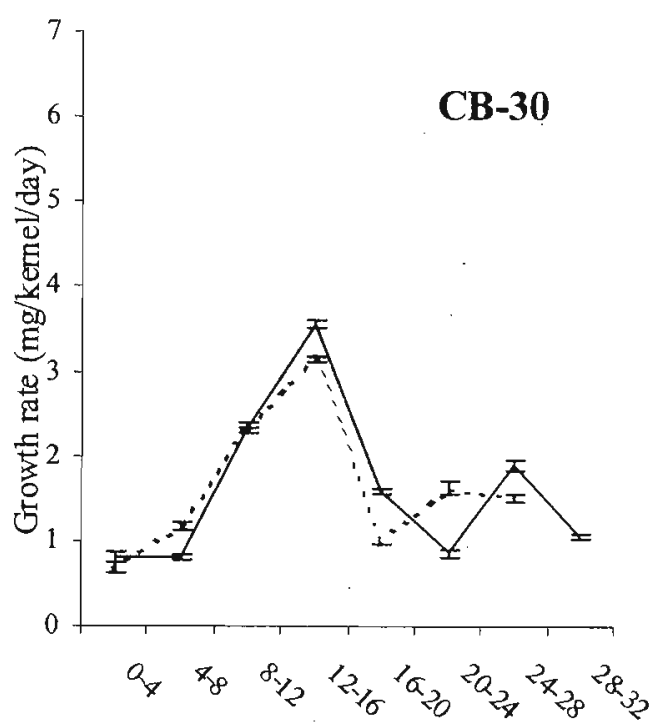

Days after anthesis
Soluble sugar content in kernel: Soluble sugar content in kernel at different days after anthesis varied between normal condition and heat stress conditions in all wheat genotypes (Figure 5). Under normal growing condition, total soluble sugar was higher during early stages, declined with increasing demand for starch synthesis up to middle part of kernel growth and thereafter increased at later stages in both $\mathrm{HT}$ and HS genotypes. Under heat stress condition, though the sugar content was lower throughout the whole

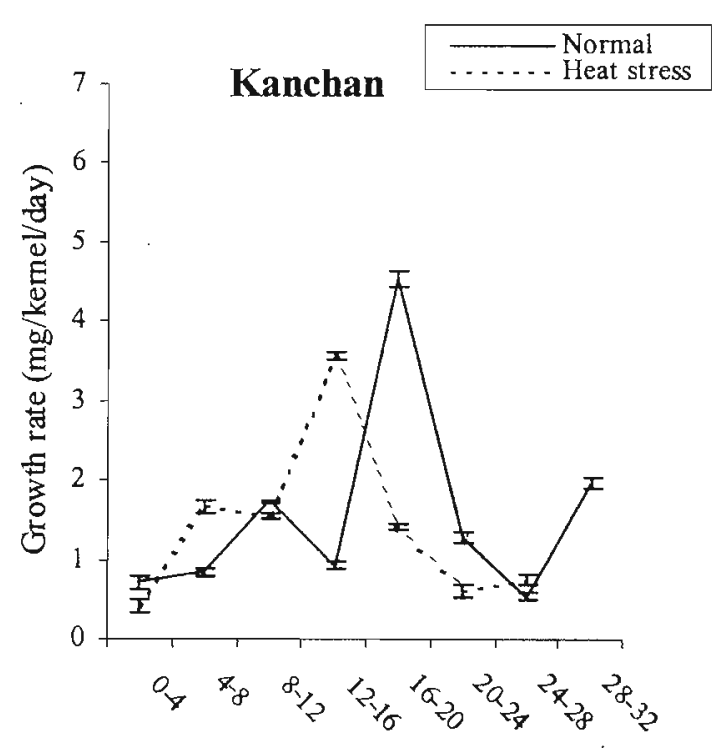

Days after anthes is

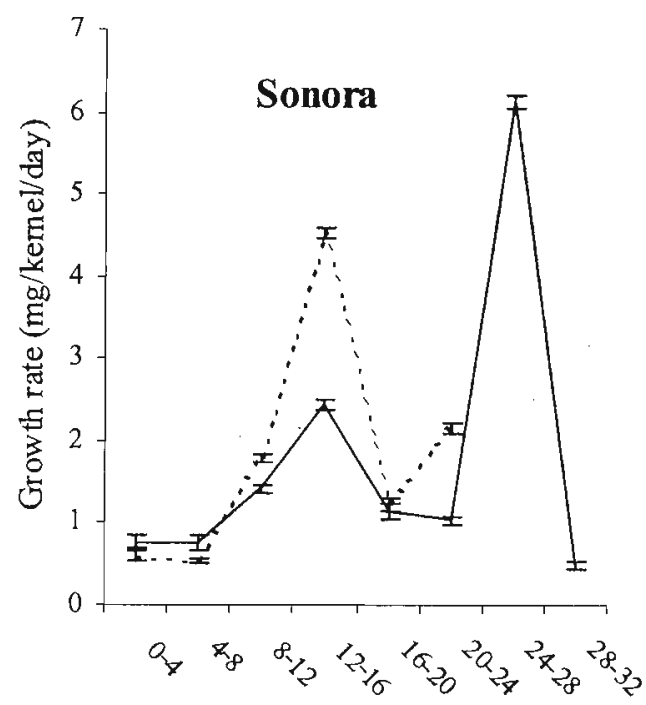

Days after anthesis

Figure 3: Kernel growth rate in different wheat genotypes at different days after anthesis under normal and post anthesis heat stress conditions. 

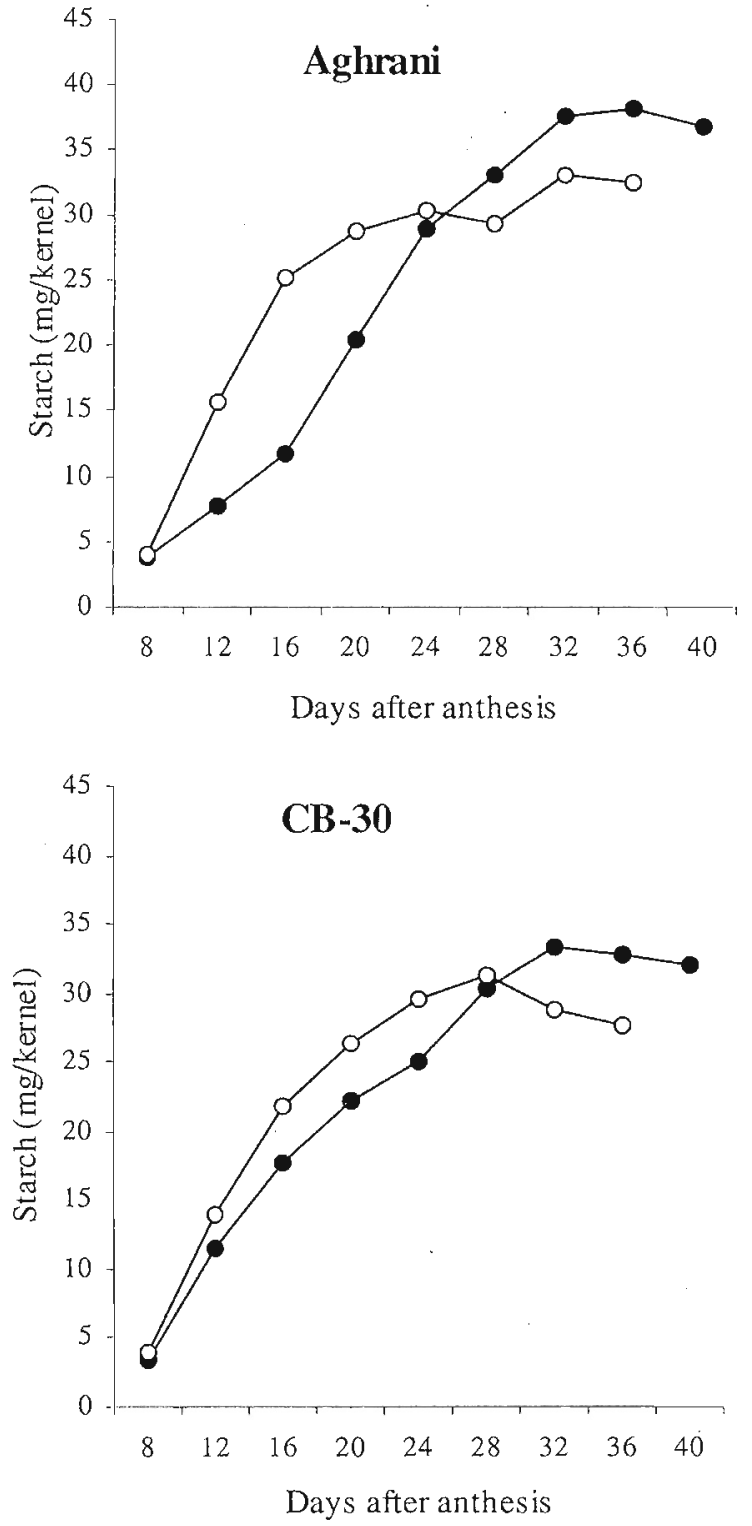
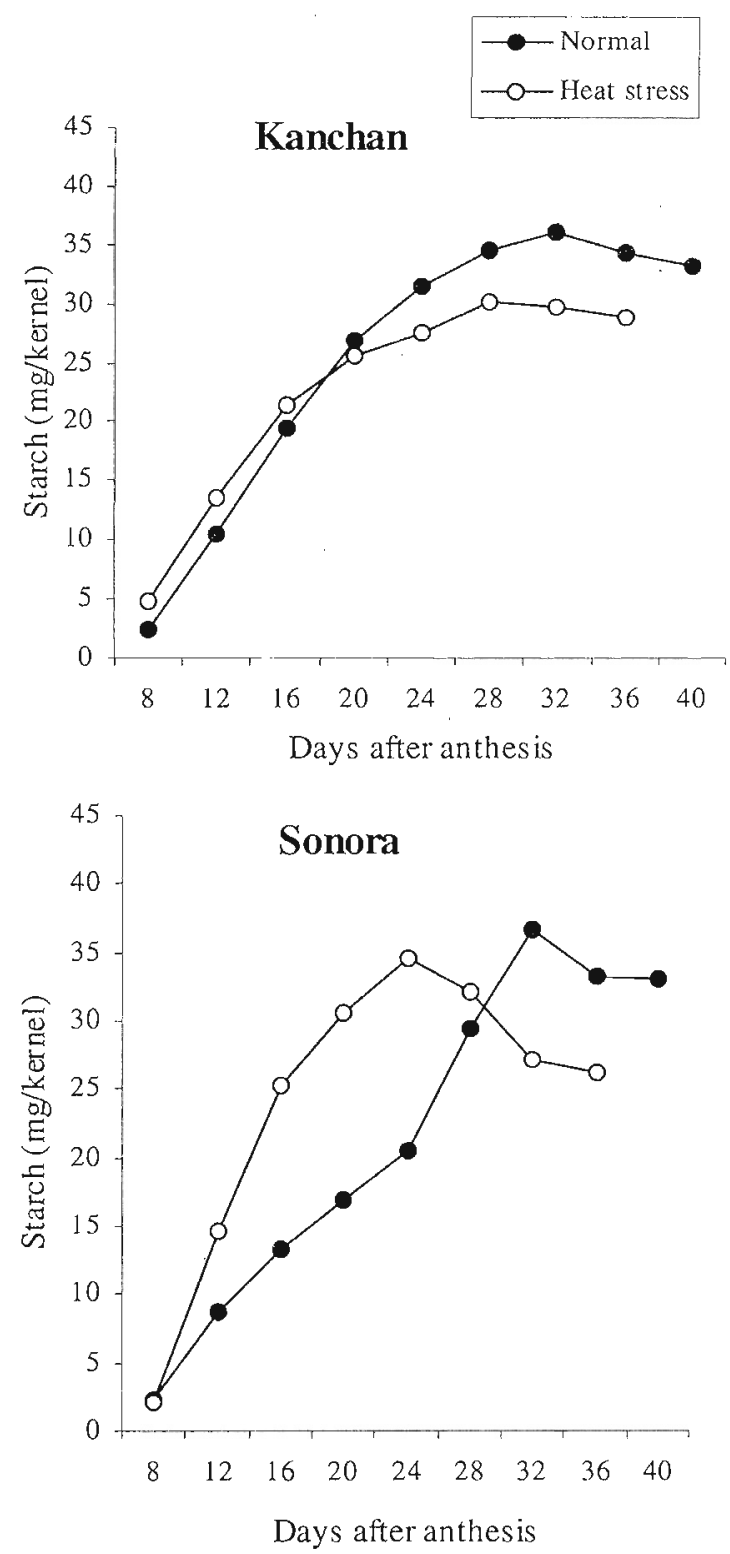

Figure 4: Starch content in wheat genotypes at different days after anthesis under normal and post anthesis heat stress conditions.

kernel developing period, the HT genotypes Aghrani, Kanchan and CB-30 showed similar trend as under normal condition. On the other hand, in HS genotype Sonora, sugar content/ kernel under heat stress condition was lower during early stages, increased with increasing growth up to $24 \mathrm{DAA}$ and thereafter declined sharply with the start of maximum rate of dry matter accumulation. A similar trend of soluble sugar content in wheat was also reported by Chanda et al. (1999). ${ }^{8}$ The results revealed that the availability of soluble sugar might become the limiting factor during the rapid kernel-growing period in all genotypes under normal condition. In heat stress condition although the HT genotypes showed a similar phenomenon as under normal condition, the HS genotype showed a different pattern of soluble sugar content in kernel. In kernel of HS genotype the early decline of dry matter accumulation and rapid kernel growth rate together resulted in the rapid increase in soluble sugar under heat stress condition. This happened apparently due to limited conversion of sugar into starch rather than limited supply of sugar in kernel. One of the speculations might be a limited enzymatic function involved in sugar to 
starch conversion step; but this needs further experimentation to confirm. However, many authors reported that regulation of starch synthesis in the cereal endosperm has been suggested to be at glucose pyrophosphorylase step $^{10}$ or at the sucrose synthase step. ${ }^{18}$ At elevated temperature, Macleod and Dufus $(1988)^{17}$, Denyer et al. $(1994)^{9}$ and Jenner $(1994)^{14}$ found reduced activity of sucrose synthase in wheat and Lafta and James $(1995)^{16}$ in Potato tubers.
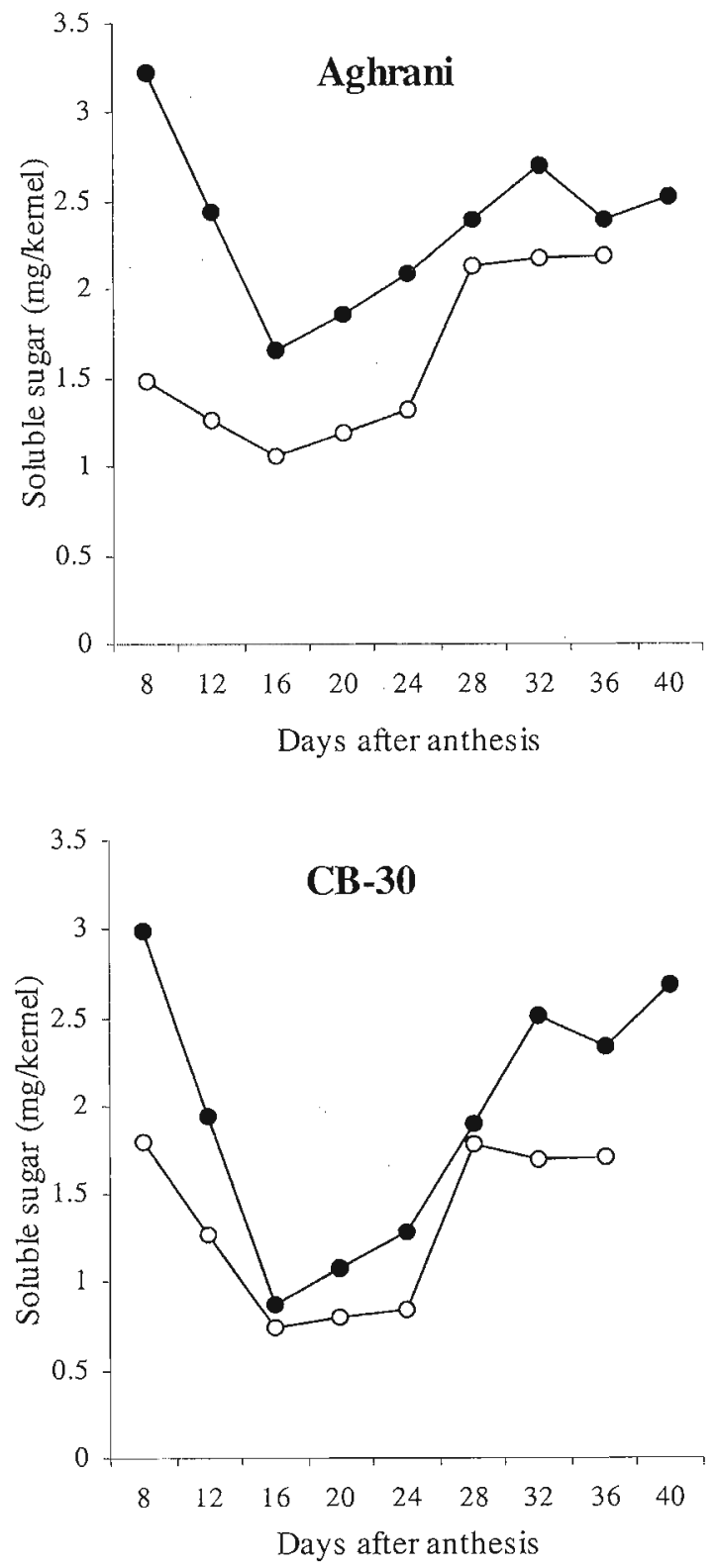

Kernel size: Individual kernel size differed significantly due to the combined effect of growing conditions and wheat genotypes (Table 1). Under normal growing condition, maximum kernel size was recorded in Aghrani (41.37 mg/kernel) which was statistically equal with that of Kanchan (41.20 $\mathrm{mg} /$ kernel). CB-30 attained the lowest kernel size (38.04 mg/kernel) which followed the kernel size of Sonora ( $39.41 \mathrm{mg} / \mathrm{kernel})$. These deviations in kernel size among the wheat genotypes were due to their genetic variation. At post anthesis heat stress condition, kernel size reduced significantly
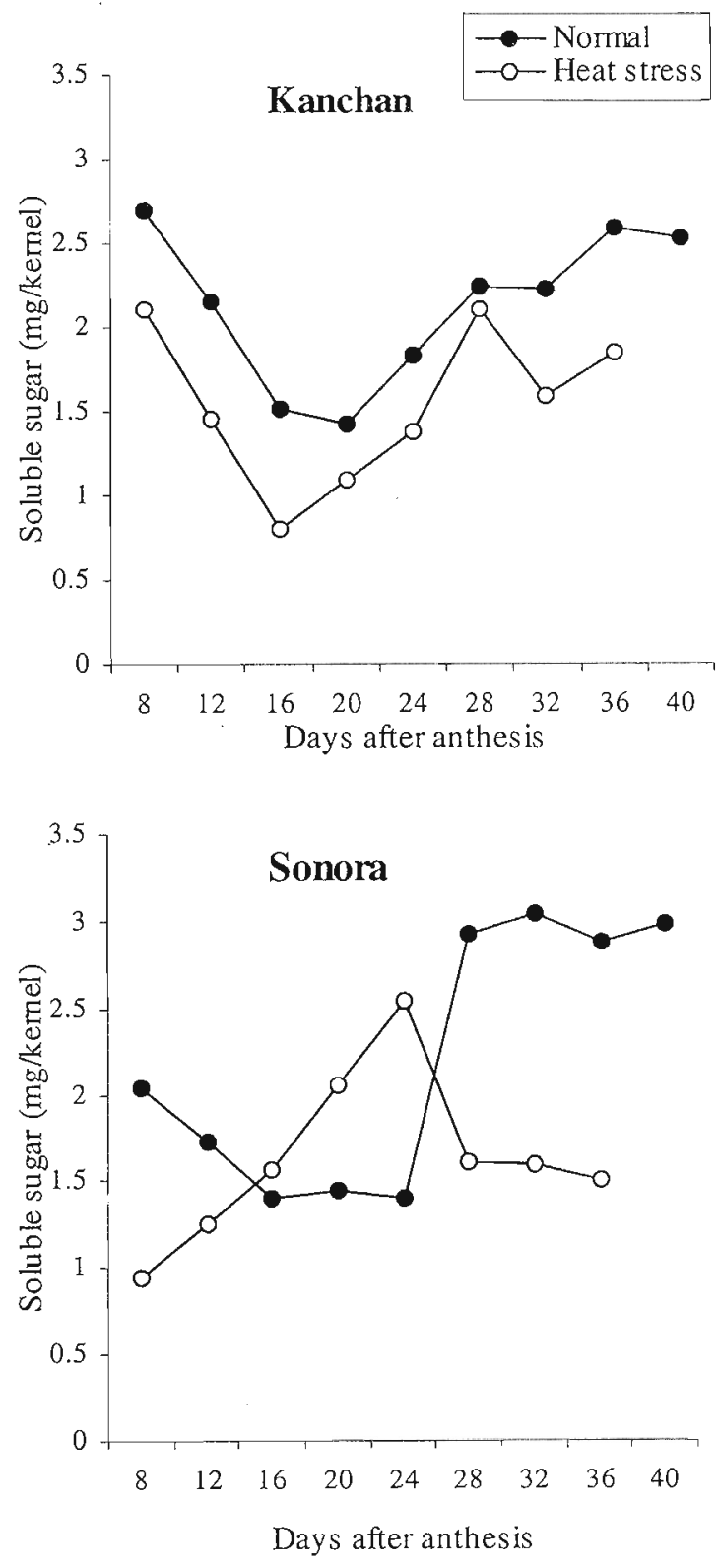

Figure 5: Soluble sugar content in wheat genotypes at different days after anthesis under normal and post anthesis heat stress conditions. 


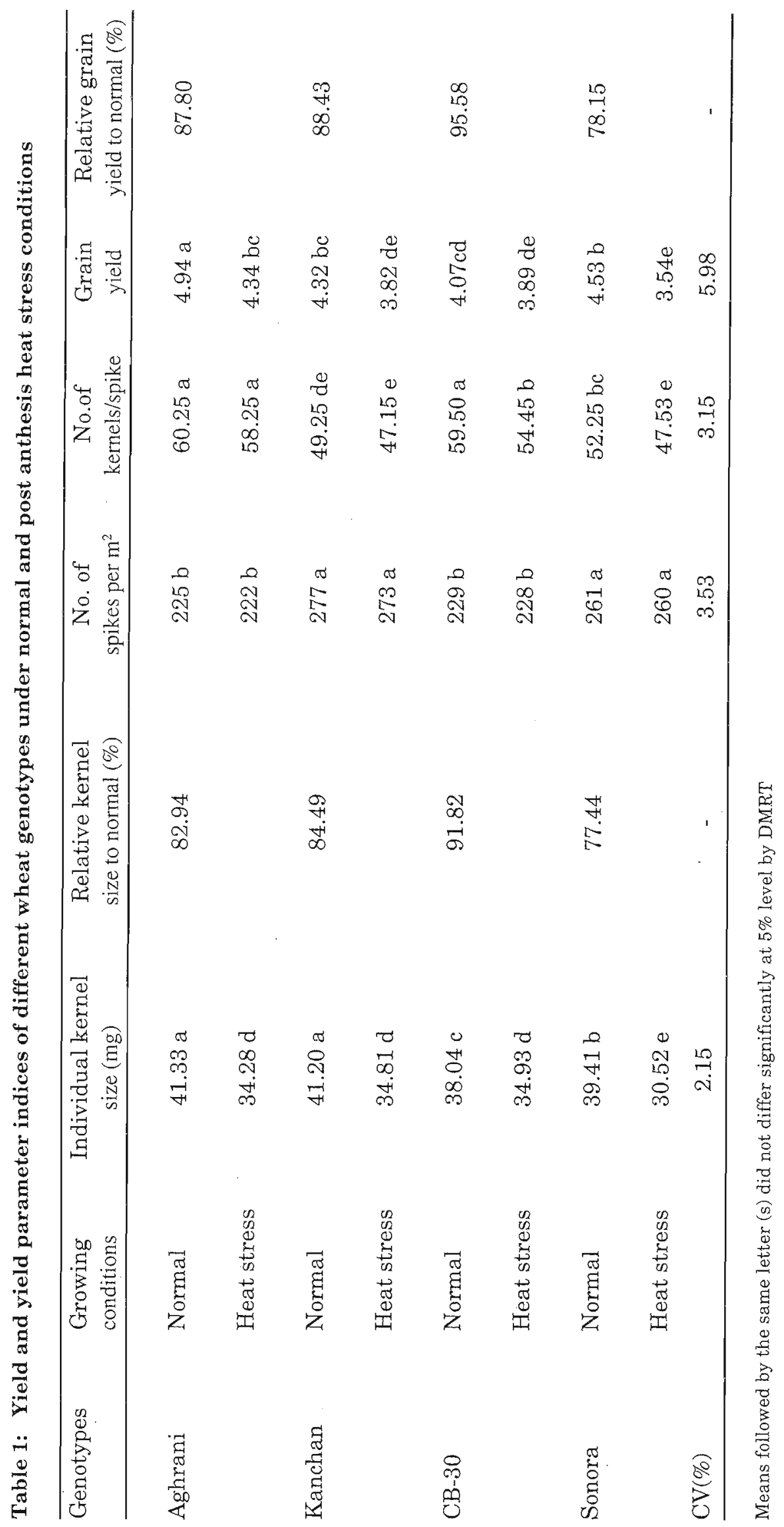


in all wheat genotypes. But the magnitude of reduction was different in different genotypes (Table 1). In this condition, the HS genotype (Sonora) attained lower relative kernel size to normal $(77.44 \%$ ) than the HT genotypes Aghrani (82.94\%), Kanchan (84.49\%) and CB-30 (91.82\%). Reduced kernel size under heat stress condition might be due to the reduction in rapid kernel growth duration. Results also suggested that reduction in kernel size was also contributed by the impairment of the process involved in sugar to starch conversion due to heat stress.

Number of spikes/ $/ \mathrm{m}^{2}$ : Number of spikes $\mathrm{m}^{-2}$ was not influenced significantly by the interaction effect of growing conditions and genotypes but differed significantly among genotypes (Table 1). Kanchan had the highest spikes $\mathrm{m}^{-2}$ (273 to 277) which was statistically similar to that of Sonora (260 to 261). Aghrani had the lowest spikes $\mathrm{m}^{-2}$ (222 to 225) which was at par with CB-30 (228 to 229). The insignificant interaction between growing conditions and wheat genotypes indicated that the differences in number of spikes $\mathrm{m}^{-2}$ among the genotypes were mainly genetically controlled and wheat plants did not suffer from heat stress in GS1 phase (emergence to double ridge) in the present study, in which tillering was determined.

Results from other studies showed that spike number $\mathrm{m}^{-2}$ was not influenced by late sowing condition. ${ }^{20} \mathrm{He}$ and Rajaram (1993) ${ }^{11}$ reported that the ear number $\mathrm{m}^{-2}$ was less sensitive while yield, grains per ear, biomass and plant height were more sensitive to heat.

Kernels per spilse: Kernel number per spike was influenced significantly by the interaction effect of growing conditions and genotypes (Table 1). Under normal growing condition, Aghrani had the highest number of kernels per spike (60.25) which was statistically similar to that of CB-30 (59.50) and Kanchan had the lowest kernels per spike (49.25). In the case of Sonora, it was the second lowest (52.25). On the other hand, at post anthesis heat stress condition, this number was reduced in all genotypes though the reduction was significant only for CB-30 and Sonora. Based on magnitude of reduction in kernels per spike, the HS genotype Sonora was affected more (9.3\%) than the HT genotypes Aghrani (3.3\%), Kanchan $(4.3 \%)$ and $\mathrm{CB}-30(8.5 \%)$. Reduced number of kernels per spike of different magnitudes were also observed by several other workers under late sowing or high temperature conditions compared to optimum sowing temperature. . $^{2,5,11,13}$

Grain yield: Growing conditions and wheat genotypes interacted significantly to govern the grain yield (Table 1). Under normal growing condition, Aghrani attained the highest grain yield $(4.94 \mathrm{t} / \mathrm{ha}$ ) which was followed by Sonora (4.53 t/ha) and Kanchan (4.32 t/ha) while CB-30 gave the lowest grain yield $(4.07 \mathrm{t} / \mathrm{ha})$. Post anthesis heat stress conditions decreased the grain yield significantly in all wheat genotypes but the degree of reduction was not equal in different genotypes. Percent relative yield to normal $(78.15 \%)$ was lower in heat sensitive Sonora and was higher in the heat tolerant Aghrani (87.80\%), Kanchan (88.43\%) and CB-30 (95.58\%). In the present study it was found that grain yield reduced by about 2.6 to $5.8 \%$ for each $1^{\circ} \mathrm{C}$ rise in average mean air temperature from normal growing conditions during anthesis to maturity in HT genotypes but it was about $7.2 \%$ in heat sensitive Sonora (Figure 1 and Table 1 ).

Reduced number of kernels per spike and reduced kernel size were the major factors responsible for reducing the grain yield under heat stress condition in the present experiment. Other yield attributes e.g., spike number $\mathrm{m}^{-2}$ was not affected by high temperature (Table 1). Results from other studies showed that late sowing caused lower grain yield in wheat compared to optimum sowing ${ }^{5,13}$. It was suggested that high relative grain yield which was the result of stable and/or long duration of photosynthetic activity under heat stress conditions could be used as a selection criterion for heat tolerance of wheat genotypes. ${ }^{2}$

From the overall results it may be concluded that reduced kernel size in heat sensitive genotype under post anthesis heat stress conditions was due to the reduction in rapid kernel growth duration and this was mainly because of poor metabolism of soluble sugars to starch rather than low availability of soluble sugar in the plant. 


\section{References}

1. Acevedo E., Nachit M. \& Ferrana G. O. (1991). Effects of heat stress on wheat and possible selection tools for use in breeding for tolerance. In: Wheat for the non-traditional warm areas (Ed D. A. Saunders). Pp. 401-420.CIMMYT, Mexico.

2. Al-Khatib K. \& Paulsen G. M. (1990). Photosynthesis and productivity during high temperature stress of wheat genotypes from major world regions. Crop Science 30: 1127-1132.

3. Asana R. D. \& Saini A. D. (1962). Studies in physiological analysis of yield. v. Grain development in wheat in relation to temperature, soil moisture and changes with age in the sugar content of the stem and in the photosynthetic surface. Indian Journal of Plant Physiology 5: 12871.

4. Asana R. D. \& Williams R. F. (1965). The effect of temperature stress on grain development in wheat. Australian Journal of Agricultural Research 16: 1-3.

5. Bhatta M. R., Hernandez J. E. \& Lates J. S. (1994). Possibilities of selecting wheat with fast grain filling rate for warmer areas. In: Wheat in Heatstressed Environments: Irrigated, Dry Areas and Rice-wheat Farming System (Eds D. A. Saunders and G.P. Hatel). Pp. 375-378. CIMMYT, Mexico.

6. Bruckner P. L. \& Frohberg R. C. (1987). Rate and duration of grain filling in spring wheat. Crop Science 27: 451-455.

7. Campbell C. A. \& Read D. W. L. (1968). Influence of air temperature, light intensity and soil moisture on the growth, yield and some growth analysis characteristics of chioiolo wheat grown in the growth chamber. Canadian Journal of Plant Science 48: 299-311.

8. Chanda S., Narmada V. K. \& Singh Y. D. (1999). Dry matter accumulation and associated changes in biochemical parameters during wheat grain development. Journal of Agronomy \& Crop Science 182: 153-159.

9. Denyer K., Hylton C. M. \& Smith A. M. (1994). The effect of high temperature on starch synthesis. Austrailian Journal of Plant Physiology 21: 783-789.
10. Dickinson D. B. \& Preiss J. (1969). Presence of ADP-glucose pyrophosphorylase in shrunken-2 and brittle-2 mutants of maize endosperm. Plant Physiology 44: 1058-1062.

11. He Z. H. \& Rajaram S. (1993). Differential responses of bread wheat characters to high temperature, Euphytica 72: 197-203.

12. Ishag H. M. \& B. A. Mohamed. (1996). Phasic development of spring wheat and stability of yield and yield components in hot environments. Field Crops Research 46: 169-176.

13. Islam N., Ahmed S. M., Razzaque M. A., Sufian A. \& Hossain M. A. (1993). A study on the effect of seeding dates on the yield of wheat varieties. Bangladesh Journal of Agricultural Research 18(1):102-107.

14. Jenner C. F. (1994). Starch synthesis in the kernels of wheat under high temperature conditions. Austrailian Journal of Plant Physiology 21: 857-867.

15. Jhala G. K. \& Jadon B. S. (1989). Variability in wheat for grain grow th rate under timely and late sowing. Gujarat Agricultural University Research Journal 15: 21-29.

16. Lafta M. A. \& James H. L. (1995). Effect of high temperature on plant growth and carbohydrate metabolism in Potato. Plant Physiology 109: 637643.

17. MacLeod L. C. \& Duffus C. M. (1988). Reduced starch content and sucrose synthase activity in developing endosperm of barley plants grown at elevated temperatures. Austrailian Journal of Plant Physiology 15: 367-375.

18. Murata T. (1974). Enzymic mechanism of starch synthesis in plants. Japan Agricultural Research Quarterly 8: 127-132.

19. Sarkar C. K.G., Srivastava P.S. L. \& Deshmukh P. S. (2001). Grain growth rate and heat susceptibility index: traits for breeding genotypes tolerant to terminal high temperature stress in bread wheat. Indian Journal of Genetics and Plant Breeding 61(3): 209-212.

20. Sikder S., Ahmed J. U., Hossain T., Miah M. A. K. \& Hossain M. M. (1999), Membrane 
thermostability, grain growth and contribution of pre-anthesis stem reserve to grain weight under late seeded condition. Thailand Journal of Agricultural Science 32(4): 465-473.

21. Sofield I., Evans L. T. \& Wardlaw I. F. (1977). Factors influencing the rate and duration of grain filling in wheat. Australian Journal of Plant Physiology 4: 785-797.

22. Stone P. J. \& Nicholas M. E. (1988). Comparison of sudden heat stress with gradual exposure to high temperature during grain filling in two varieties differing in heat tolerance. II. Fractional protein accumulation. Austrailian Journal of Plant Physiology 25: 1-11.

23. Tashiro T. \& Wardlaw I. F. (1989). A comparison of the effect of high temperature on grain development in wheat and rice. Annals of Botany 64:95-65.
24. Tashiro T. \& Wardlaw I. F. (1990). The effect of high temperature at different stages of ripening on grain set, grain weight and grain dimensions in the semi-dwarf wheat "Banks". Annals of Botany 65(1): 51-61.

25. Wiegand C. L. \& Cuellar J. A. (1981). Duration of grain filling and kernel weight of wheat as affected by temperature. Crop Science 21: 95-101.

26. Yoshida S., Forno D. A., Cock J. H. \& Gomez K. A. (1976). Laboratory Manual for Physiological Studies of rice (Third edition). The International Rice Research Institute, Los Banos, Laguna, Philippines. Pp. 46-49.

27. Hossain M. M., Takeda H. \& Senboku T. (1995). Improved method of determination of membrane thermostability for screening heat-tolerant and sensitive varieties of Brassica. Japan International Research Centre for Agricultural Sciences Journal 2(1): 19-27. 